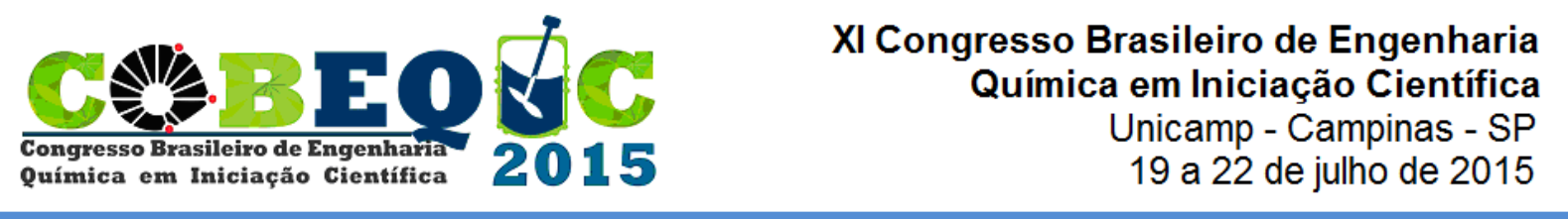

\title{
REOLOGIA DO ESCOAMENTO DO SANGUE EM ARTÉRIA
}

\author{
M. G. PEREIRA ${ }^{1}$, R. A. MALAGONI ${ }^{2}$ e J. R. D. FINZER ${ }^{2,3}$ \\ ${ }^{1}$ Universidade Federal de Uberlândia, Faculdade de Medicina \\ ${ }^{2}$ Universidade Federal de Uberlândia, Faculdade de Engenharia Química \\ ${ }^{3}$ Universidade de Uberaba, Curso de Engenharia Química \\ E-mail para contato: mguisoni@gmail.com
}

\begin{abstract}
RESUMO - O sangue é uma suspensão constituída por uma fase aquosa contínua (plasma) contendo sais, açúcares, proteínas e uma fase de particulados que incluem os eritrócitos (glóbulos vermelhos), leucócitos (glóbulos brancos do sangue) e plaquetas. O plasma é um fluido newtoniano, com uma viscosidade entre 1,16 e $1,35 \mathrm{cP}$ a $37^{\circ} \mathrm{C}$. $\mathrm{O}$ sangue apresenta um comportamento nãonewtoniano. No trabalho é descrita a fenomenologia do escoamento do sangue. A tensão aplicada é modelada com o uso da lei de Potência. A viscosidade aparente do sangue foi obtida pela aplicação de modelo que considera os nutrientes. Efetuando-se um balanço de forças na aorta, obtém-se a tensão na parede. $\mathrm{Da}$ literatura a vazão de sangue pela aorta é de $5 \mathrm{~L} / \mathrm{min}$, isso possibilita o cálculo da tensão na parede igual a $326 \mathrm{~Pa}$. Do balanço de forças calcula-se a perda de pressão na aorta em $2608 \mathrm{~Pa}$, a velocidade máxima do sangue no centro da aorta, $0,75 \mathrm{~m} / \mathrm{s}$ e o gradiente de velocidade em $60 \mathrm{~s}^{-1}$. Segue-se o cálculo da viscosidade aparente do sangue igual a $5,43 \mathrm{mPa} \cdot \mathrm{s}$. Mostra-se no trabalho que ao aumentar a velocidade devido a uma diminuição do diâmetro da aorta, a viscosidade aparente irá diminuir assim como a tensão na parede, minimizando as influências físicas sobre a artéria aorta. Este trabalho indica, fenomenologicamente, o comportamento do sangue ao escoar pela aorta e serve de subsídio para explicar, o porquê na ocorrência de obstruções parciais, a influência nas paredes é amenizada.
\end{abstract}

\section{INTRODUÇÃO}

Jean Leonard Marie Poiseuille (1797 - 1869) foi um físico e fisiologista francês, natural de Paris. Estudou durante um ano na École Plytechnique, em Paris e em 1828 com um trabalho denominado Recherches sur la vigor du coeur aortique recebeu o grau D.Sc. Gotthilf Heinrich Ludwig Hagen (1797 - 1884), natural de Konigsberg, Alemanha; o qual atuou como físico e engenheiro hidráulico, em 1839, publica um artigo sobre o escoamento de água em tubos cilíndricos, cujos resultados eram parecidos com os de Poiseuille, embora estes fossem mais extensos e acurados. Poiseuille queria estabelecer uma relação funcional entre quatro variáveis: a taxa de escoamento volumétrico de água destilada em um tubo; a diferença de pressão no tubo; o comprimento e o diâmetro do tubo (Sutera e Skalak, 1993).

Do trabalho desses pesquisadores, originou-se uma expressão matemática para mensurar a taxa volumétrica de escoamento em regime laminar de fluidos em tubos circulares conhecida como equação de Hagen-Poiseuille (Equação 1). O regime laminar se caracteriza pela não mistura das camadas do fluido em escoamento (Bird et al., 2002). A equação de 


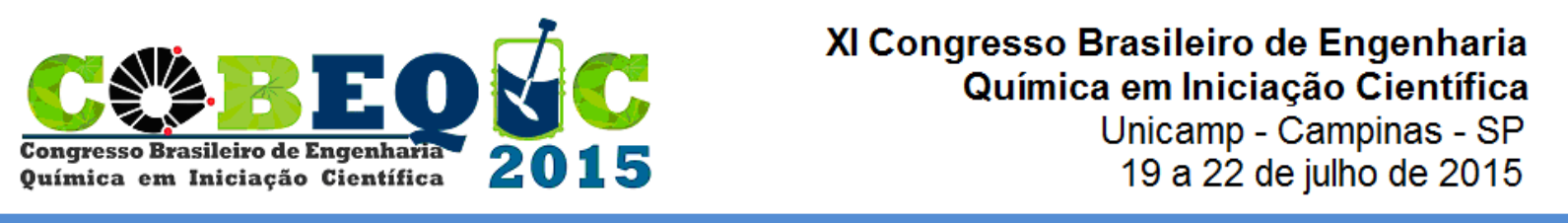

Hagen-Poiseuille, Equação 1, válida para o regime laminar, é usada para quantificar a vazão do fluido em escoamento em um tubo (Fraiha et al., 2010).

$$
Q=\frac{\pi \cdot \Delta P \cdot R^{4}}{8 \cdot \mu \cdot L}
$$

sendo: $\mathrm{Q}$ a vazão $\left(\mathrm{m}^{3} / \mathrm{s}\right) ; \Delta \mathrm{P}$ a perda de pressão $(\mathrm{Pa}) ; \mathrm{R}$ o raio $(\mathrm{m}) ; \mathrm{L}$ o comprimento $(\mathrm{m}) ; \mu$ a viscosidade (Pa.s).

\section{FLUÍDOS NÃO NEWTONIANOS: REOLOGIA DO SANGUE}

O sangue é uma suspensão constituída por uma fase aquosa contínua (plasma) contendo sais, açúcares, proteínas e uma fase discreta, que incluem os eritrócitos (glóbulos vermelhos), que constituem a vasta maioria (mais de 99,5\%) dos particulados, leucócitos (glóbulos brancos do sangue) e plaquetas. O plasma é um fluido Newtoniano, com uma viscosidade entre 1,16 e $1,35 \mathrm{cP}$ a $37^{\circ} \mathrm{C}$. Ao contrário do plasma, o sangue tem um comportamento nãonewtoniano, principalmente devido à presença de glóbulos vermelhos, conforme mostra a Figura 1.

Figura 1 - Representação esquemática do sangue e seus constituintes

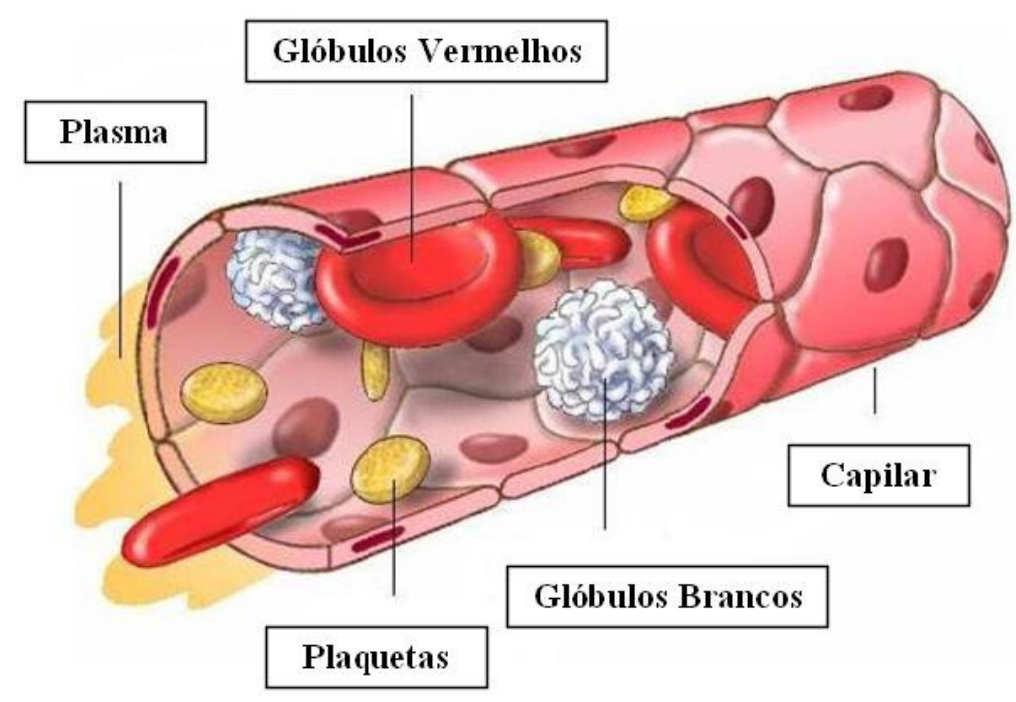

\section{MATERIAL E MÉTODOS}

A tensão aplicada ao sangue em escoamento modelada com lei da Potência (Equação 2).

$$
\tau=k \cdot(d v / d r)^{r}
$$

$\tau$ é a tensão aplicada; $k$ o fator de consistência e $n$ o fator de comportamento do sangue. 
O comportamento dos fluidos é pode ser destacado conforme mostra a Figura 2, destacando-se a curva da Lei de Potência (acima da linha verde, fluido newtoniano) que rege o comportamento do sangue (pseudoplástico).

Figura 2 - Comportamento de fluidos não-newtonianos (a reta verde consiste no comportamento newtoniano)

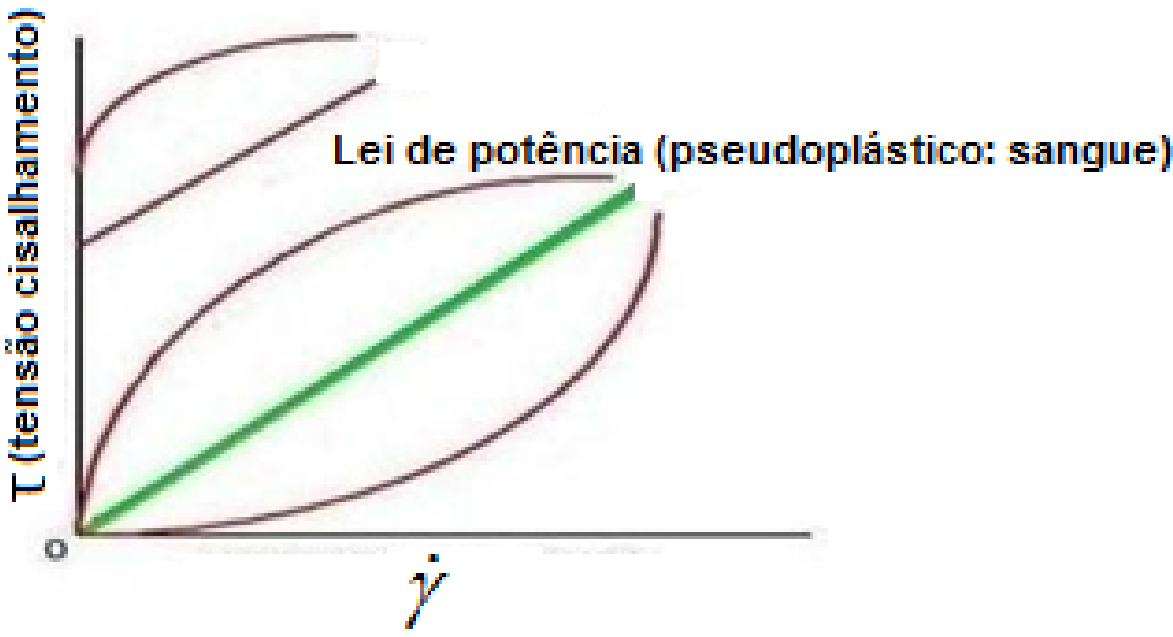

Sendo: $\quad \dot{\gamma}=d v / d r$ a derivada da velocidade do sangue em relação à posição radial.

(Skelland, 1967), mostra que a a viscosidade aparente é quantificada pela Equação (3).

$$
\mu_{\text {aparente }}=k \dot{\gamma}^{n-1}
$$

Conforme mostrado por Santos (2009), os fatores de comportamento do fluido e de consistência podem ser correlacionados de acordo com as Equações (4), (5) e (6), o que possibilita o cálculo da viscosidade aparente $\mathrm{em} \mathrm{mPa} \cdot \mathrm{s}$.

$$
\begin{aligned}
& k=c_{1} e^{c_{2} h} \\
& n=c_{3} \cdot h \\
& c_{1}=c_{1}^{*} e^{\left(c_{4} \cdot P / h^{2}\right)}
\end{aligned}
$$


Sendo: $C_{1}^{*}=0,00797(\mathrm{~Pa} \cdot \mathrm{s})^{\mathrm{n}} ; C_{2}=17,167 ; \mathrm{C}_{3}=0,651 ; \mathrm{C}_{4}=0,00795(\mathrm{~Pa} \cdot \mathrm{s})^{\mathrm{n}}$

$\mathrm{P}$ consiste na diferença da proteína total no sangue menos a albumina, na situação normal igual a 0,0325 e h refere-se ao hematócrito, ou seja, a porcentagem total de hemácias em relação ao plasma, sendo seu valor médio de $45 \%$.

\subsection{Tensão na Parede dos Vasos e Esquema do Coração}

Efetuando-se um balanço de forças em um vaso sanguíneo, obtém-se a tensão na parede em função da perda de pressão por atrito $\left(\Delta \mathrm{P}_{\mathrm{f}}\right)$, conforme a Equação 7.

$$
\tau_{\mathrm{w}}=\mathrm{D} \cdot \Delta \mathrm{P}_{\mathrm{f}} / 4 \cdot \mathrm{L}
$$

A velocidade em uma posição radial no vaso sanguíneo é quantificada pela Equação 8.

$$
v=\left[\frac{\Delta P_{f}}{2 \cdot k \cdot L}\right]^{1 / n} \cdot \frac{n}{n+1}\left[R^{\frac{n+1}{n}}-r^{\frac{n+1}{n}}\right]
$$

Sendo: L e R o comprimento e o raio do vaso; $r$ consiste na distância do centro do vaso até um ponto genérico (ver a Figura 3 ).

Figura 3 - Detalhes de escoamento em um vaso

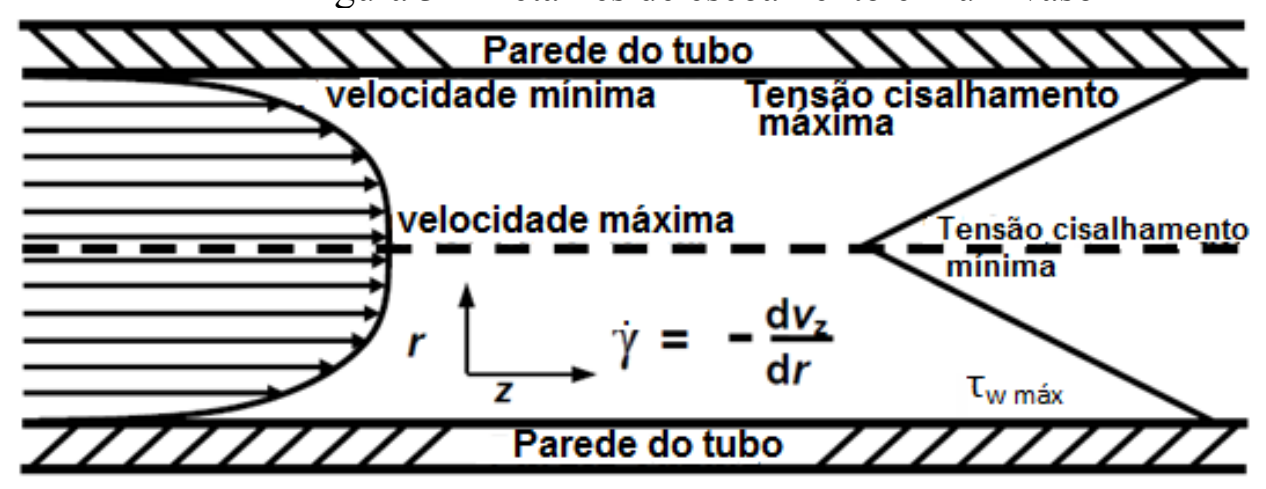

A vazão do fluido não-newtoniano que segue o modelo da Potência é quantificada pela Equação (9).

$$
\frac{Q}{\pi \cdot R^{3}}=\frac{n}{3 \cdot n+1}\left(\frac{\tau_{w}}{k}\right)^{1 / n}
$$


A Figura 4 situa a aorta no coração a qual possui comprimento e diâmetros médios de 0,05 e $0,025 \mathrm{~m}$, respectivamente.

Figura 4 - Representação esquemática dos principais orifícios do coração

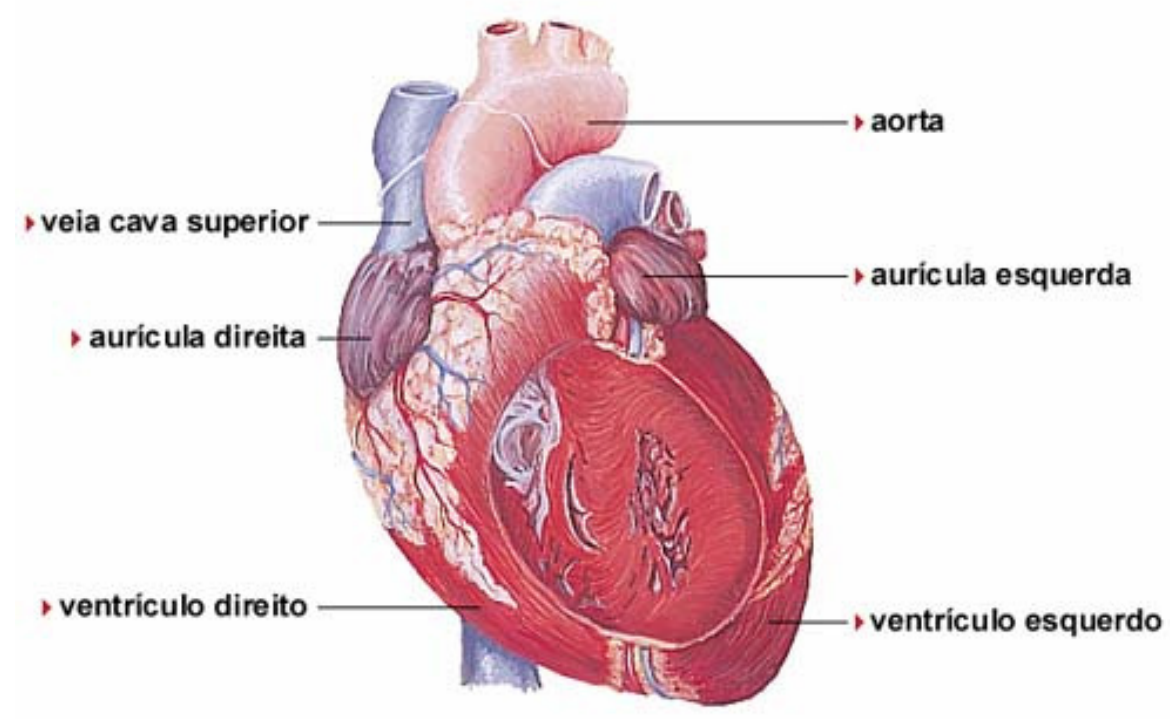

\section{RESULTADOS E DISCUSSÃO}

A viscosidade do sangue pode ser então determinada utilizando o valor padrão de vazão do sangue pela artéria aorta de $5 \mathrm{~L} / \mathrm{min}$. Ao utilizar as as Equações (4), (5) e (6), obtém-se: n $=0,70705 \mathrm{e} \mathrm{k}=18,035 \mathrm{~Pa} \cdot \mathrm{s}^{0.70705}$.

Utilizando-se a Equação 9, obtém-se o valor para a tensão na parede da artéria aorta, sendo $\tau_{w}=326 \mathrm{~Pa}$.

Do balanço de forças calcula-se a perda de pressão na aorta pela Equação 7 como sendo:

$\Delta \mathrm{P}=2608 \mathrm{~Pa}$. A velocidade do sangue no centro da aorta pode ser determinada pela Equação 8, como: $u_{\operatorname{máx}}=0,75 \mathrm{~m} / \mathrm{s}$. Portanto, o gradiente de velocidade entre o centro e a e a parede da artéria pode ser calculado, considerando o fato que para $\mathrm{r}=\mathrm{R}$ (raio da aorta) a velocidade do sangue é nula.

$$
\frac{d u}{d r}=\frac{0-u_{\max }}{0,0125-0}=-60 \mathrm{~s}^{-1}
$$

A Equação 3 possibilita o cálculo da viscosidade aparente:

$$
\mu_{\text {aparente }}=18,0035 \cdot 60^{0,70705-1}=5,43 \mathrm{mPa} \cdot \mathrm{s}
$$


Efetuando-se uma simulação, se a velocidade no centro do vaso aumentar devido a uma diminuição do diâmetro, por problemas de depósitos ou alterações nas paredes (ver a Figura 5), como redução em $10 \%$ do diâmetro do vaso, obtém-se com procedimento similar ao anterior: $\tau_{w}=427 P a$.

Figura 5 - Vaso sanguíneo parcialmente obstruído

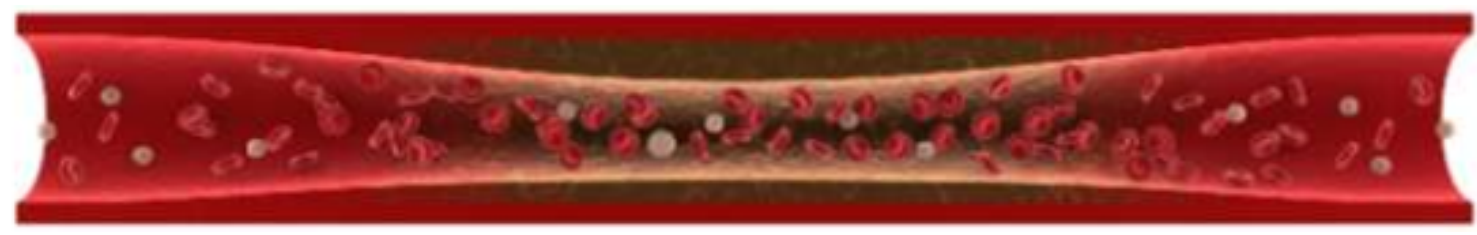

A Equação 8 possibilita obter: $u_{\text {máximo }}=0,97 \mathrm{~m} / \mathrm{s}$, e o uso da Equação 3 é possibilita o cálculo da viscosidade aparente do sangue para a situação simulada, sendo: $\mu_{\text {aparente }}=4,90 \mathrm{mPa} \cdot \mathrm{s}$. Portanto: A viscosidade aparente irá diminuir, minimizando o efeito do aumento da tensão na parede do vaso sanguíneo.

\section{CONCLUSÕES}

Este trabalho indica, fenomenologicamente, o comportamento do sangue ao escoar pela aorta e serve de subsídio para explicar, o porquê na ocorrência de obstruções parciais, a influência no escoamento é minimizada devido à diminuição da viscosidade do sangue ao aumentar a tensão de cisalhamento. A viscosidade do sangue variou de 5,43 mPa.s para 4,90 mPa.s na ocorrência de $10 \%$ de obstrução do diâmetro do vaso .

\section{REFERÊNCIAS}

BIRD, R.B., STEWART, W. E, e LIGHTFOOT, E .N. Fenômenos de Transporte, $2^{\mathrm{a}}$ edição, Rio de janeiro: LTC, 2004. 838 p.

FRAIHA, M., BIAGI, J. D., FERRAZ, A. C. O., SVERZUT, C. Projeto e construção de reômetro capilar para caracterização de alimentos para animais. 1998 Fraiha et al. Ciência Rural, v.40, n.9, set, 2010. Ciência Rural, v.40, n.9, p.1998-2004, 2010.

SANTOS, N. A. A. Estudo da reologia de fluidos análogos ao sangue. Porto: Universidade do Porto. Fundação para a Ciência e Tecnologia (FCT), 2009. 85 p.

SKELLAND, A. H. P. Non-newtonian flow and heat transfer. New York: John Wiley. 1967. $469 \mathrm{p}$.

SUTERA, S. P.; SKALAK, R. The history of Poiseuille's law. Annu. Rev. Fluid Mech., v. 25, p. 1-19, 1993. 